\title{
SHIFTED PLANE PARTITIONS OF TRAPEZOIDAL SHAPE
}

\author{
ROBERT A. PROCTOR
}

\begin{abstract}
The number of shifted plane partitions contained in the shifted shape $[p+q-1, p+q-3, \ldots, p-q+1]$ with part size bounded by $m$ is shown to be equal to the number of ordinary plane partitions contained in the shape $(p, p, \ldots, p)$ ( $q$ rows) with part size bounded by $m$. The proof uses known combinatorial descriptions of finite-dimensional representations of semisimple Lie algebras. A separate simpler argument shows that the number of chains of cardinality $k$ in the poset underlying the shifted plane partitions is equal to the number of chains of cardinality $k$ in the poset underlying the ordinary plane partitions. The first result can also be formulated as an equality of chain counts for a pair of posets. The pair of posets is obtained by taking order ideals in the other pair of posets.
\end{abstract}

1. Introduction and results. Let $p \geq q$ be two positive integers (see Figure 1). A shifted plane partition $S$ contained in the shifted shape $[p+q-1, p+q-3, \ldots, p-q+1]$ ( $q$ rows) with part size bounded by $m$ is a collection of nonnegative integers $S_{i j}, 1 \leq$ $i \leq q, i \leq j \leq p+q-i$, such that $S_{i j} \geq S_{i+1, j}, S_{i, j} \geq S_{i, j+1}$ and $S_{i j} \leq m$. An (ordinary) plane partition $R$ contained in the shape $(p, p, \ldots, p)$ ( $q$ rows) with part size bounded by $m$ is a collection of nonnegative integers $R_{i j}, 1 \leq i \leq q, 1 \leq j \leq p$, satisfying the same conditions. The main result of this paper is

THEOREM 1. The number of shifted partitions contained in the shifted shape $[p+q-1, p+q-3, \ldots, p-q+1]$ with part size bounded by $m$ is equal to the number of ordinary plane partitions contained in the shape $(p, p, \ldots, p)(q$ rows) with part size bounded by $m$.
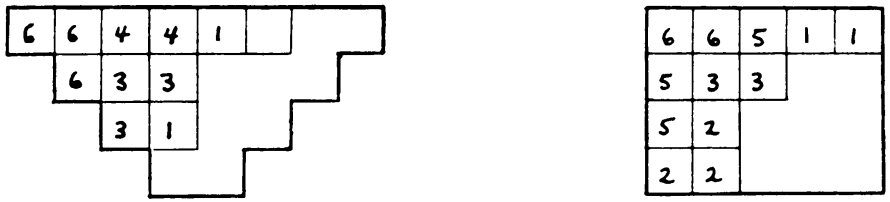

$$
\mathrm{p}=5, \mathrm{q}=4, \mathrm{~m}=6 .
$$

FiguRE 1. Shifted and ordinary plane partitions.

There is a well-known expression for the second quantity [Mac, Example I.5.13(b)].

Received by the editors November 4, 1982 and, in revised form, February 28, 1983.

1980 Mathematics Subject Classification. Primary 05A17; Secondary 17B10.

Key words and phrases. Plane partitions, zeta polynomials, Young tableaux, representations of symplectic Lie algebras. 
COROLlaRY. The number of shifted plane partitions contained in the shifted shape $[p+q-1, p+q-3, \ldots, p-1+1]$ with part size bounded by $m$ is

$$
\prod_{i=1}^{q} \prod_{j=1}^{p} \prod_{k=1}^{m} \frac{(i+j+k-1)}{(i+j+k-2)}
$$

It has long been known that the ordinary plane partitions of Theorem 1 describe weights of a certain irreducible representation of the Lie algebra $\operatorname{sl}(p+q, \mathbf{C})$. More recently, combinatorial descriptions of weights of irreducible representations of $s p(2 k, \mathbf{C})$ have geen given. These combinatorial descriptions can be easily reformulated in terms of shifted plane partitions. The shifted plane partitions of Theorem 1 can then be seen to correspond to the weights of a collection of irreducible representations of $s p(p+q, \mathbf{C})$ when $p+q$ is even. (A combinatorial argument can be used to adjust to the case of $p+q$ being odd.) A branching rule is a description of the decomposition of an irreducible representation of a Lie algebra into irreducible representations of a subalgebra. Branching rules have been studied extensively in the physics literature over the past 15 years. We will use a branching rule for $s p(2 k, \mathbf{C}) \hookrightarrow s l(2 k, \mathbf{C})$ due to King [Ki1] to decompose the irreducible representation of $s l(p+q, \mathbf{C})$ into the desired collection of irreducible representations of $s p(p+q, \mathbf{C})$. A combinatorial description of the Littlewood-Richardson coefficients together with a combinatorial argument are needed to apply this branching rule to the situation at hand.

Set $a=p-1$ and $b=q-1$. Let $T_{a b}$ ( $T$ for trapezoid) denote the region of the integral cartesian plane with coordinates $(x, y)$ where $0 \leq y \leq b, x \geq y$ and $x \leq a+b-y$. The extreme points of this region are $(0,0),(a+b, 0),(a, b)$ and $(b, b)$. Let $P_{a b}(P$ for product) denote the rectangular region in the plane with extreme points $(0,0),(a, 0),(a, b)$ and $(0, b)$. Give each set the usual partial order from $\mathbb{N} \times \mathbb{N}$ : The maximal elements of $T_{a b}$ are $(a+b, 0),(a+b-1,1), \ldots,(a, b)$ and the unique maximal element of $P_{a b}$ is $(a, b)$. Fix $m>0$ and consider a sequence of order ideals $I_{1} \subseteq I_{2} \subseteq \cdots \subseteq I_{m}$ in $T_{a b}\left(P_{a b}\right)$. Next to each element of $T_{a b}\left(P_{a b}\right)$, write down the number of ideals $I_{j}$ in which that element occurs. It is clear that this procedure describes a one-one correspondence between the shifted (ordinary) plane partitions of Theorem 1 and increasing sequences $I_{1} \subseteq I_{2} \subseteq \cdots \subseteq I_{m}$ in $T_{a b}\left(P_{a b}\right)$.

Given any poset $P$, let $J(P)$ denote the lattice of order ideals of $P$ (ordered by inclusion). Increasing sequences $I_{1} \subseteq I_{2} \subseteq \cdots \subseteq I_{m}$ of order ideals in $P$ become multichains of total cardinality $m$ in $J(P)$, or $m$-multichains. For any poset $P$, let $\zeta(m+1)$ be the number of $m$-multichains in $P$. In general, the function $\zeta(n)$ is a polynomial in $n$, called the zeta polynomial [Ede]. Theorem 1 states that the zeta polynomials of $J\left(T_{a b}\right)$ and $J\left(P_{a b}\right)$ are equal.

This discussion provides the backdrop for our second result.

THEOREM 2. The number of m-multichains in $T_{a b}$ is equal to the number of m-multichains in $P_{a b}$.

This result was also recently obtained by John Stembridge. Our proof uses a simple telescoping sum argument together with an existing combinatorial formula. The zeta polynomial of a product of two posets is the product of their zeta polynomials, and the zeta polynomial of the total order on $c+1$ elements is $\left(\begin{array}{c}n-1+c \\ c\end{array}\right)$. 
COROLlaRY. The number of m-multichains in $T_{a b}$ is

$$
\left(\begin{array}{c}
m+a \\
a
\end{array}\right)\left(\begin{array}{c}
m+b \\
b
\end{array}\right) .
$$

Theorem 2 states that the zeta polynomials of $T_{a b}$ and $P_{a b}$ are equal. Thus the zeta polynomials of $T_{a b}$ and $P_{a b}$ coincide in addition to the coincidence of zeta polynomials of $J\left(T_{a b}\right)$ and $J\left(P_{a b}\right)$. While it may be possible to convert our proof of Theorem 2 to a bijective proof, the question of a combinatorial correspondence for Theorem 1 seems to be a complete mystery. Furthermore, we know of no theoretical considerations which would lead one to suspect the truth of one of these theorems when told of the truth of the other.

The author is indebted to Richard Stanley for news of the empirical discovery of Theorems 1 and 2 for $p=q \leq 4$. Stanley was considering these posets because this author had conjectured that the number of maximal chains in the weak Bruhat order on the hyperoctahedral group of rank $n+1$ was equal to the number of extensions of $T_{n n}$ to a total order, i.e. the number of shifted standard Young tableaux on the corresponding shape. On the other hand, Stanley himself had conjectured that the number of maximal chains was equal to the number of order extensions of $P_{n n}$, viz. the number of ordinary standard Young tableaux on a square shape. It is easy to use the hook and shifted hook formulas [Mac, Examples I.5.2, III. 7.8] to show that the number of order extensions of $P_{a b}$ is always equal to the number of order extensions of $T_{a b}$. (This is the equality of the highest coefficients of the zeta polynomial identity of Theorem 1.) The poset $T_{n n}$ is the order dual of the poset of positive roots of type $B_{n+1}$ (and of type $C_{n+1}$ also). Further empirical work has shown that the poset $T_{n n}$ is probably the right poset to consider for the hyperoctahedral problem. Stanley has solved the maximal chain problem for the weak Bruhat order on the symmetric group [Sta]. His proof uses Schur functions, which are characters of representations of the special linear group. The representations of symplectic groups viewpoint used here may be useful for the solution of the hyperoctahedral maximal chain problem.

2. Proof of Theorem 1. Denote the fundamental weights of a simple Lie algebra by $\omega_{1}, \omega_{2}, \ldots, \omega_{n}$. Let $X_{n}(\omega)$ denote the finite-dimensional irreducible representation of highest weight $\omega$ of the simple complex Lie algebra of type $X$ and rank $n$. The expression "indexes the weights" will be short for "indexes the weights-with-multiplicities". 7.1].

The following fact is well known [Boe, Theorem 5.3] or [Pro, proof of Proposition

Lemma 1. Plane partitions contained in the shape $(p, p, \ldots, p)$ ( $q$ rows) with parts $\leq m$ index the weights of the representation $A_{p+q-1}\left(m \omega_{q}\right)$.

Given an $n$-tuple of nonnegative integers $\mathbf{m}=\left(m_{1}, m_{2}, \ldots, m_{n}\right)$, let $\lambda=\left(\lambda_{1}, \ldots\right.$, $\left.\lambda_{n}\right), \lambda_{1} \geq \lambda_{2} \geq \cdots \geq \lambda_{n} \geq 0$, be the partition of $\sum_{i=1}^{n} m_{i} i$ with $m_{i}$ columns of length $i$; equivalently, $\lambda_{j}=\sum_{i=j}^{n} m_{i}$. If $\mathbf{m}$ and $\lambda$ correspond in this fashion, and if $\omega=\sum_{i=1}^{n} m_{i} \omega_{i}$, then we will also refer to the representation $C_{n}(\omega)$ by $C_{n}(\lambda)$.

Given a shifted plane partition $S$ with $k$ rows, let $\mu_{i}=S_{i, i}$. Note that $\mu_{1} \geq \mu_{2} \geq$ $\cdots \geq \mu_{k}>0$. Call $\mu$ the profile partition of $S$. 
LEMMA 2. Shifted plane partitions contained in the shifted shape $[2 n, 2 n-2, \ldots, 2]$ with profile $\lambda$ index the weights of $C_{n}(\lambda)$.

Proof. The tableaux of [Ki2, p. 496] corresponding to $C_{n}(\lambda)$ have shape $\lambda$. Change $\overline{1}, 1, \overline{2}, \ldots, n-1, \bar{n}, n$ to $2 n, 2 n-1,2 n-2, \ldots, 3,2,1$. The entries are now strictly decreasing along the columns ( $x$-direction) and weakly decreasing along the rows ( $y$-direction), and an entry in the $i$ th row does not exceed $2 n-2 i+2$ (see Figure 2). Convert this (ordinary) plane partition to a solid Ferrers diagram, slide the dots in plane $x=i$ up $i-1$ places in the $z$-direction, and project to the $x z$ plane. The resulting shifted plane partition has profile $\lambda$. The process clearly reverses. This lemma can also be proved using [Bac].
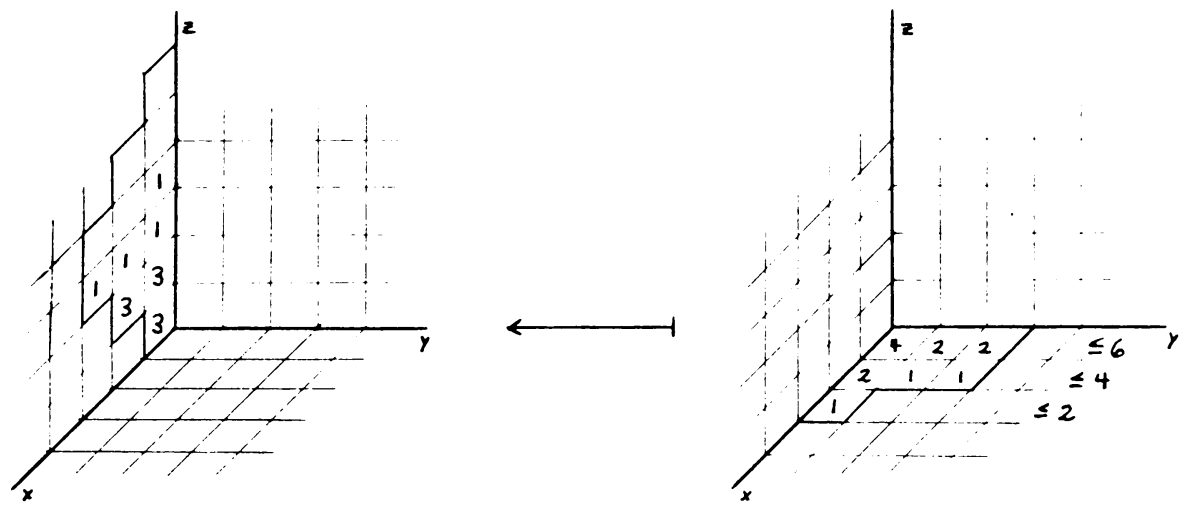

Figure 2. Lemma 2: trapezoidal shifted plane partitions

from column strict plane partitions with row bounds.

LEMMA 3. If $p+q-1$ is even, the shifted plane partitions contained in the shape $[p+q-1, p+q-3, \ldots, p-q+1]$ ( $q$ rows $)$ with parts $\leq m$ index the weights of the representation $\bigoplus C_{n}(\lambda)$, where $2 n=p+q-1$ and the direct sum is over all $\lambda$ with $q$ or fewer parts, no part exceeding $m$. If $p+q-1$ is odd, the shifted plane partitions contained in the shape $[p+q-1, p+q-3, \ldots, p-q+1]$ with parts $\leq m$ index the weights of the representation $\bigoplus C_{n}(\lambda)$, where $2 n=p+q$ and the direct sum is over all $\lambda$ with $q$ or fewer parts, no part exceeding $m$, the length of each column of $\lambda$ having the same parity as $q$.

Proof. The first statement follows directly from Lemma 2 and the definition of profile. Apply Lemma 2 to the direct sum of the second statement to obtain a collection of shifted plane partitions contained in the shape $[p+q, p+q-2, \ldots, p-$ $q+2]$. Remove the entry $S_{i, i}$ from the beginning of each row. View a shifted plane partition as parallel layers of dots stacked up, each layer a shifted Ferrers diagram. The number of rows occupied in a layer is equal to the length of the corresponding column of the profile partition. The operation above removes the leftmost dot from each row in each layer. If a shifted plane partition had profile $\lambda$ before the operation, then each column length of the new profile $\lambda^{\prime}$ is the same or one less than the old column length (see Figure 3). The column lengths of $\lambda$ are all even or all odd, depending upon the parity of $q$. Therefore all such possible (column lengths must weakly decrease) profiles $\lambda^{\prime}$, and all shifted plane partitions with those profiles, arise exactly once each from the set of all shifted plane partitions with the old profile $\lambda$. Then as the direct sum runs over all profile partitions with column lengths of the 
same parity as $q$, the above operation will produce all profile partitions with $\leq q$ parts, each part $\leq m$, exactly once each. The new shifted plane partitions with these profiles occur at most once each, have parts $\leq m$, and are contained in the shape $[p+q-1, p+q-2-1, \ldots, p-q+2-1]=[p+q-1, p+q-3, \ldots, p-q+1]$. It is clear that all such shifted plane partitions are produced by this process.

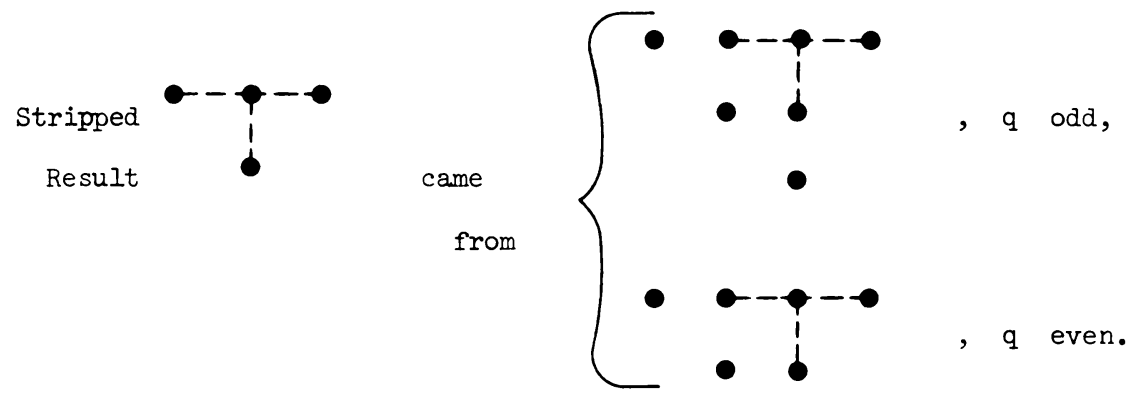

Figure 3. Lemma 3: typical $k$ th layer, $1 \leq k \leq m$.

LEMMA 4. Under the inclusion $\operatorname{map} s p(2 n, \mathbf{C}) \hookrightarrow \operatorname{sl}(2 n, \mathbf{C})$,

$$
A_{2 n-1}\left(m \omega_{r}\right)=\bigoplus C_{n}\left(\sum_{i=0}^{r} m_{i} \omega_{i}\right) \quad(r \leq n),
$$

where the direct sum is over all $\mathbf{m}$ such that $\sum_{i=0}^{r} m_{i}=m$ and $m_{i} \neq 0$ implies that $i$ is of the same parity as $r$. (Set $\omega_{0}=0$, the highest weight of the trivial representation.) Embed $\operatorname{sl}(2 n, \mathbf{C})$ into the upper left hand corner of $\operatorname{sl}(2 n+1, \mathbf{C})$. Then under the composed embedding $s p(2 n, \mathbf{C}) \hookrightarrow \operatorname{sl}(2 n+1, \mathbf{C})$,

$$
A_{2 n}\left(m \omega_{r}\right)=\bigoplus C_{n}\left(\sum_{i=0}^{r} m_{i} \omega_{i}\right) \quad(r \leq n),
$$

where the direct sum is over all $\mathbf{m}$ such that $\sum_{i=0}^{r} m_{i}=m, 0 \leq i \leq r$.

Proof. We have associated a partition $\lambda$ to each irreducible representation $C_{n}(\omega)$. To each partition $\lambda$, one can associate a Schur function $s_{\lambda}$. Given an irreducible representation of $A_{2 n-1}$, King's branching rule [Ki1, Equation 4.7] produces a sum of skew Schur functions $s_{\lambda / \beta}$, indexed by skew partitions $\lambda / \beta$ [Mac, p. 4]. Upon expressing these skew Schur functions as positive integral linear combinations of ordinary Schur functions, one finds the partitions $\lambda$ which occur in the direct sum $\bigoplus_{\lambda} C_{n}(\lambda)$ decomposition of the original representation. The skew partitions produced for $A_{2 n-1}\left(m \omega_{r}\right)$ are all those of the form $\mu / \beta$, where $\mu=(m, m, \ldots, m)$ ( $r$ rows), and $\beta$ has all columns of even length. (To use King's rules, one associates a partition $\lambda$ to $A_{n}(\omega)$ in the same manner we have been using for $C_{n}(\omega)$.) From the combinatorial descriptions of Schur and skew Schur functions [Mac, Equation I.5.12], it is obvious that skew Schur functions whose shapes have straight bottom and right boundaries are just the Schur functions corresponding to the ordinary shapes obtained by rotating the skew shapes $180^{\circ}$ in the plane. Thus the first part of the lemma is obtained rather easily.

Similar reasoning produces the branching rule

$$
A_{2 n}\left(m \omega_{j}\right)=\bigoplus_{k+l=m} A_{2 n-1}\left(l \omega_{j-1}+k \omega_{j}\right)
$$


from [Ki1, Equation 4.5]. Use [Ki1, Equation 4.7] again. Now decompose the skew Schur functions $s_{\mu / \beta}$, where $\mu=(m, m, \ldots, m, k)$ is an $r$-tuple, $0 \leq k \leq m$, and $\beta$ has all columns of even length. Fix $k$, and let $r \geq \beta_{1}^{*} \geq \beta_{2}^{*} \geq \cdots \geq \beta_{m}^{*} \geq 0$ be the column lengths of $\beta$. Let $g$ be the number of $\beta_{i}^{*}$ equal to $r$.

CLAIM. $s_{\mu / \beta}=\sum_{\lambda} s_{\lambda}$, where the sum is over all shapes $\lambda$ with column lengths $r-1-\beta_{m}^{*}+\delta_{m} \geq r-1-\beta_{m-1}^{*}+\delta_{m-1} \geq \cdots \geq r-1-\beta_{1}^{*}+\delta_{1} \geq 0$, where exactly $k-g$ of the $\delta_{i}$ are 1 and exactly $l+g$ are 0 .

Use the Littlewood-Richardson rule [Mac, Equations I.9.1, I.9.2] (see Figure 4). Scanning $\mu / \beta$ in Semitic fashion, insert positive integers in the boxes of this shape in such a way that at any step the number of $i+1$ 's inserted so far does not exceed the number of $i$ 's inserted, and such that the entries weakly increase to the right and strictly increase down the columns. Since the right boundary for the first $r-1$ rows is straight, within these rows the $i$ th entry in a column must be an $i$. The entries in the last row are any $k-g$ nonzero numbers chosen from $\left\{r-\beta_{1}^{*}, r-\beta_{2}^{*}, \ldots, r-\beta_{m}^{*}\right\}$ and placed in weakly increasing order. Slide boxes with entry $i$ up to the $i$ th row and then left justify each row of boxes. According to Littlewood-Richardson, the sum of the Schur functions on these shapes is the desired decomposition. For fixed $\beta$ and $k$, the possible choices of the $\delta_{i}$ correspond exactly to the choices of entries for the last row of boxes in $\mu / \beta$.

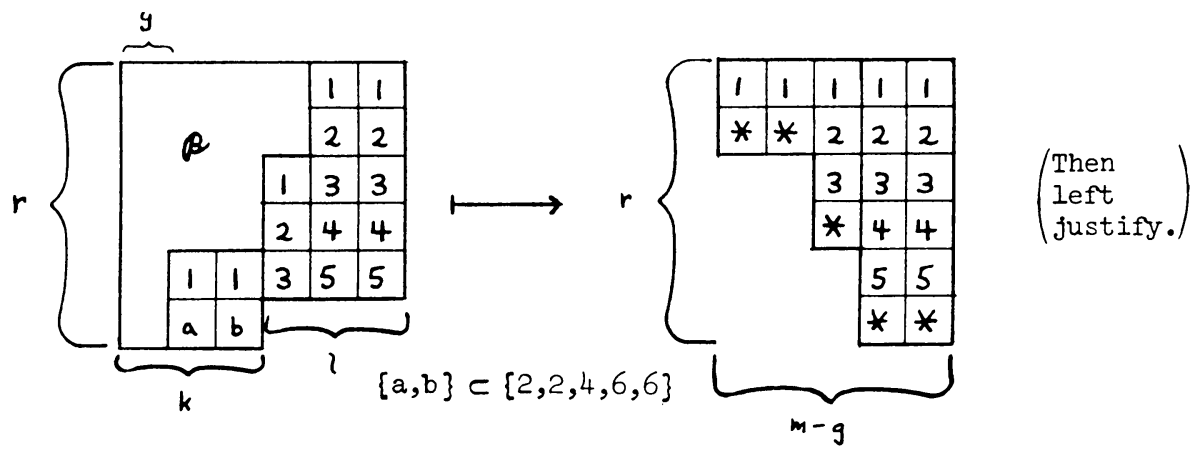

Figure 4. Lemma 4: applying Littlewood-Richardson to $\mu / \beta$.

To complete the proof of the second part of the lemma, note that as $\beta$ runs over all partitions with columns of even length, the partitions $\lambda$ produced in the claim will exactly exhaust all partitions with $\leq r$ parts, each part $\leq m$.

To prove Theorem 1 when $p+q-1$ is odd, choose $2 n=p+q, r=q$, and combine the first part of Lemma 4 with Lemma 1 and the second part of Lemma 3 . If $p+q-1$ is even, choose $2 n=p+q-1, r=q$, and combine the second part of Lemma 4 with Lemma 1 and the first part of Lemma 3. The proof of Theorem 1 is complete, by the equality of representation vector space dimensions.

3. Proof of Theorem 2. Fix $a \geq b \geq 0$ throughout. Let $E_{d}(m)$ be the number of $m$-multichains in $T_{a b}$ whose maximal elements have $y$-coordinate $d$, where $0 \leq d \leq$ $b$. Let $F_{u, v}(m)$ be the number of $m$-multichains in $P_{u v}$ with all elements lying on or below the line $x=y$. By [Car, Equation 2.9] or [Ges],

$$
F_{u, v}(m)=\left(\begin{array}{c}
m+u \\
u
\end{array}\right)\left(\begin{array}{c}
m+v+1 \\
v
\end{array}\right)-\left(\begin{array}{c}
m+u+1 \\
u+1
\end{array}\right)\left(\begin{array}{c}
m+v \\
v-1
\end{array}\right)
$$


and

$$
\begin{aligned}
E_{d}(m) & =F_{a+b-d, d}(m)-F_{a+b-d, d-1}(m) \\
& =\left(\begin{array}{c}
m+a+b-d \\
a+b-d
\end{array}\right)\left(\begin{array}{c}
m+d \\
d
\end{array}\right)-\left(\begin{array}{c}
m+a+b-d+1 \\
a+b-d+1
\end{array}\right)\left(\begin{array}{c}
m+d-1 \\
d-1
\end{array}\right) .
\end{aligned}
$$

Now the total number of $m$-multichains in $T_{a b}$ is

$$
\varsigma(m+1)=\sum_{d=0}^{b} E_{d}(m)=\left(\begin{array}{c}
m+a \\
a
\end{array}\right)\left(\begin{array}{c}
m+b \\
b
\end{array}\right),
$$

by collapsing the sum. But this is just the zeta polynomial for $P_{a b}$.

One can probably convert this to a bijective proof by using the nonintersecting path viewpoint of [Ges]. To apply Gessel's techniques, convert the $m$-multichains counted by $F_{u, v}(m)$ to 2-rowed column strict plane partitions with $m$ columns and with entries in the first row between 1 and $u+1$, and entries in the second row between 0 and $v$.

\section{REFERENCES}

[Bac] K. Baclawski, Character generators for unitary and symplectic groups, J. Math. Phys. (to appear).

[Boe] H. Boerner, Representations of groups, North-Holland, London, 1969.

[Car] L. Carlitz, Rectangular arrays and plane partitions, Acta Arith. 13 (1967), 29-47.

[Ede] P. Edelman, Zeta polynomials and the Möbius function, European J. Combin. 1 (1980), 335340 .

[Ges] I. Gessel, Determinants and plane partitions, European J. Combin. (to appear).

[Ki1] R. C. King, Branching rules for classical Lie groups using tensor and spinor methods, J. Phys. A 8 (1975), 429-449.

[Ki2] _ Weight multiplicities for the classical groups, Group Theoretical Methods in Physics (Janner, Janssen and Boon, eds.), Lecture Notes in Physics, No. 50, Springer-Verlag, New York, 1976.

[Mac] I. G. Macdonald, Symmetric functions and Hall polynomials, Oxford University Press, New York, 1979.

[Pro] R. Proctor, Bruhat lattices, plane partition generating functions, and minuscule representations, European J. Combin. (to appear).

[Sta] R. Stanley, On the number of reduced decompositions of elements of Coxeter groups, preprint.

Department of Mathematics, University of California, Los Angeles, CaliforNIA 90024 